Short Report

\title{
Does Lymphocyte/CRP Ratio Predict Progression of Disease in COVID-19 Patients with Myocardial Injury?
}

\author{
Umut KARABULUT ${ }^{1}$, Dilay KARABULUT ${ }^{2}$ \\ ${ }^{1}$ Acibadem International Hospital, Department of Cardiology, Istanbul, Turkey \\ ${ }^{2}$ Health Sciences University Bakırköy Dr. Sadi Konuk Training and Research Hospital, Department of Cardiology, İstanbul, \\ Turkey
}

Turk J Int Med 2021;3(Supplement 1):S25-S26 DOI: $\underline{10.46310 / \text { tjim. } 875857}$

Keywords: Covid-19, myocardial injury, lymphocyte/C-reactive protein ratio

This study aims to reveal whether the lymphocyte-C-reactive protein ratio (LCR), a systemic inflammatory marker, predicts disease progression in COVID-19 patients with myocardial injury.

A total of 172 patients, 18 years and older, hospitalized due to COVID-19 between April 2020 and May 2020 in our hospital were included retrospectively. Hemoglobin, leukocyte, lymphocyte, neutrophil, thrombocyte, AST, ALT, creatinine, LDH, albumin, ferritin, triglyceride, procalcitonin, C-reactive protein, fibrinogen, $\mathrm{D}$-dimer, troponin, LCR values of the patients were recorded. LRC ratios were amplified as $\mathrm{x} 100$. Patients were divided into two groups as with and without myocardial injury. IBM SPSS Statistics 21.0 program was used for statistical analysis. Statistically, $p<0.05$ was considered significant.

Study patients were divided into two groups as with and without myocardial injury. The patients' mean age was $68.56 \pm 13.43$ years, $58.46 \pm 16.67$ years, respectively $(p=0.002)$. There was no difference between the groups in terms of the clinical severity of the disease, the severity of lung involvement on computed tomography, coronary artery disease, diabetes mellitus, chronic obstructive pulmonary disease, those with lymphocyte, C-reactive protein, LCR, ferritin, D-Dimer, and fibrinogen (all $\mathrm{p}>0.05$ ). Hypertension (HT), chronic renal failure (CRF) were more common in the group with myocardial injury $(p<0.01, \quad p<0.01)$. Procalcitonin and creatinine levels were significantly higher in the group with myocardial injury ( $p=0.002, p<0.001)$. When we analyzed the correlation analysis of parameters associated with myocardial injury with troponin, it showed a good correlation with CRF ( $r: 0.484, p<0.001)$, moderate with procalcitonin (r: $0.274, p<0.001)$, and weak correlation with age ( $\mathrm{r}$ : $0.180, p=0.18)$ and HT (r: 0.159, p=0.37). DM and D-dimer did not correlate ( $\mathrm{r}: 0.055, \mathrm{p}=0.472$ and $\mathrm{r}$ : $0.072, p=0.345$, respectively). In the multivariate regression analysis, only CRF (Odds ratio [OR]: 11.062 (95\% confidence interval [CI]: 1.86665.580) and reference procalcitonin levels (OR: 1.183, 95\% CI: 1.014-1.379) predicted myocardial 
injury.

A recent meta-analysis evaluated laboratory test results of severe and non-severe COVID-19 cases at the time of admission. They found that lymphocytes, monocytes, eosinophils, hemoglobin, and platelet levels were significantly reduced. In contrast, high neutrophil counts were found among complete blood count indices in severe and non-severe patients. Inflammatory or infection markers (erythrocyte sedimentation rate, C-reactive protein, procalcitonin, lactate dehydrogenase, but not interleukin-6), coagulation function tests (fibrinogen, prothrombin time, and D-dimer), and glucose were positively correlated with COVID-19 severity. ${ }^{1}$ Our study found that LRC did not predict disease progression in COVID-19 patients with myocardial injury.

\section{Conflict of Interests}

Authors declare that there are none.

\section{Acknowledgment}

This study has been presented in $17^{\text {th }}$ Uludag Internal Medicine National Winter Congress, $6^{\text {th }}$ Bursa Family Medicine Association National Congress, $11^{\text {th }}$ Uludag Internal Medicine Nursing Congress, 5-7 March 2021, Bursa, Turkey.

\section{References}

1. Ghahramani S, Tabrizi R, Lankarani KB, Kashani SMA, Rezaei S, Zeidi N, Akbari M, Heydari ST, Akbari H, Nowrouzi-Sohrabi P, Ahmadizar F. Laboratory features of severe vs. non-severe COVID-19 patients in Asian populations: a systematic review and metaanalysis. Eur J Med Res. 2020 Aug 3;25(1):30. doi: 10.1186/s40001-020-00432-3. 